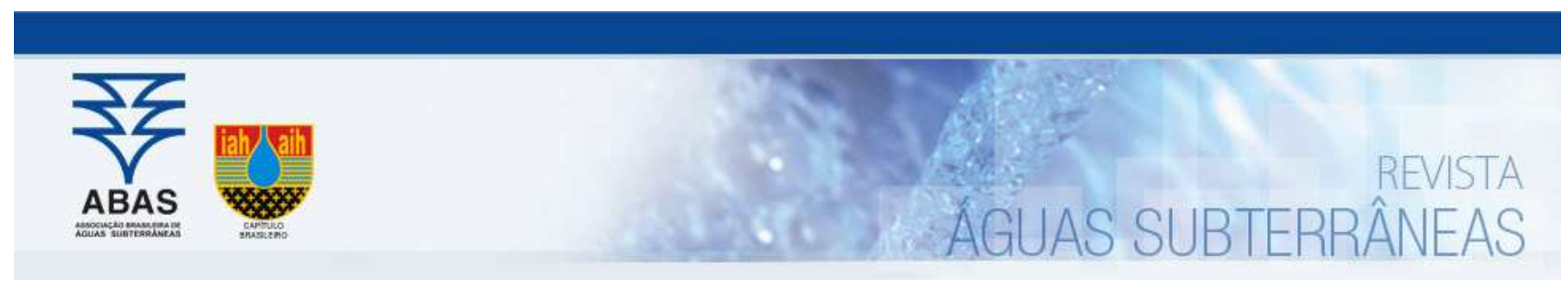

Artigos

\title{
Comparação entre índices de potencial de lixiviação para agrotóxicos utilizados na Sub-Bacia do Natuba, Vitória de Santo Antão-
} Pernambuco

\author{
Comparison between leaching potential indexes for agrochemicals used in the \\ Natuba Sub Basin, Vitória de Santo Antão-Pernambuco
}

\begin{abstract}
Jonathas Gomes de Carvalho Marques; Marília Regina Costa Castro Lyra1; Renata Maria Caminha Mendes de Oliveira Carvalho1; Rogéria Mendes do Nascimento1; José Antônio Aleixo da Silva2; Suzana Maria Gico Lima Montenegro3 ${ }^{\circledR}$
\end{abstract}

1 Instituto Federal de Pernambuco (IFPE), Recife, PE

2 Universidade Federal Rural de Pernambuco (UFRPE), Recife, PE

3 Universidade Federal de Pernambuco (UFPE), Recife, PE

$凹$ jonathasgomes@hotmail.com, marilialyra@recife.ifpe.edu.br, ren.carvalho@hotmail.com, rogeriamendes@recife.ifpe.edu.br, jaaleixo@uol.com.br, suzanam.ufpe@gmail.com

\begin{tabular}{|c|c|}
\hline & Resumo \\
\hline $\begin{array}{l}\text { Palavras-chave: } \\
\text { Modelagem matemática. } \\
\text { Avaliação de risco ambiental. } \\
\text { GUS. }\end{array}$ & $\begin{array}{l}\text { A contaminação ambiental por agrotóxicos vem sendo crescentemente abordada por diversos autores na literatura e discutida } \\
\text { pela sociedade civil, por causa de suas externalidades negativas. Este estudo visou calcular e comparar o potencial de lixivia- } \\
\text { ção e contaminação dos princípios ativos de agrotóxicos que são utilizados na sub-bacia hidrográfica do rio Natuba, município } \\
\text { de Vitória de Santo Antão-Pernambuco. Para tanto, utilizou-se índices que se propõem a avaliar a capacidade de um determi- } \\
\text { nado princípio ativo de agrotóxico lixiviar ou ser transportado no meio ambiente, a saber: Groundwater Screening Index (GSI), } \\
\text { Groundwater Ubiquity Score (GUS), Relative Leaching Potential Index (RLPI), Leaching IndeX (LIX), Leaching Index (LEACH) e } \\
\text { critério da California Departament of Food and Agriculture (CDFA). Os dados dos índices GSI, GUS, LIX, LEACH e RLPI demons- } \\
\text { traram valores semelhantes por meio da análise de variâncias, ou seja, cada índice tende a indicar, em sua faixa de valores, a } \\
\text { mesma propensão de lixiviação do agrotóxico. Assim, ao comparar os enquadramentos de cada um dos agrotóxicos, destaca- } \\
\text { ram-se, pelo risco ambiental: picloram, hexazinone, clorantraniliprole, carbofurano, thiamethoxam, 2,4 D sal dimetilamina, } \\
\text { imidacloprido, azoxistrobina, cartape, dicloreto de paraquate. Dessa forma, deve-se evitar o uso desses ingredientes ativos e } \\
\text { substituí-los por outros com menor potencial de lixiviação, visando à proteção da área. }\end{array}$ \\
\hline
\end{tabular}

Abstract

Keywords:

Environmental contamination by pesticides has been increasingly addressed by authors in the literature and by the civil media, due to their external responsibility. This study aimed to reduce the leaching and contamination potential of the main pesticides that are detectable in the Natuba river sub basin, in the city of Vitória de Santo Antão-Pernambuco. To do so, indexes that are proposed to measure the level of an environmental capacity of a system to leach or be transported to the environment were used, namely: Groundwater Screening Index (GSI), Groundwater Ubiquity Score (GUS), Relative Potential Leaching Index (RLPI), Leaching IndeX (LIX), Leaching Index (LEACH) and California Department of Food and Agriculture (CDFA) criteria. The data of the GSI, GUS, LIX, LEACH and RLPI indexes demonstrated the levels of analysis by variances, that is, each index, in its scale of values, the same propensity to leach the pesticide. Thus, when compared with agrochemicals, the environmental risk was highlighted: picloram, hexazinone, chlorantraniliprole, carbofuran, thiamethoxam, 2,4 D dimethylamine salt, imidacloprid, azoxystrobin, cartape, paraquat dichloride. Thus, the use of assets should be avoided and replaced with other ones with less leaching potential, aiming at the protection of the area.

DOI: http:/dx.doi.org/10.14295/ras.v33i1.29239

\section{INTRODUÇÃO}

A contaminação por agrotóxicos é um tema que se destacou na sociedade pela forma com que seus impactos vêm sendo observados e pela sua abordagem recorrente na literatura científica no que tange ao foco no homem e ao meio ambiente (ar, solo e recursos hídricos). O Brasil, por sua vez, sobressai-se negativamente pelo uso desses insumos com grandes arrecadações para os fa- bricantes. Marcos legais contribuíram fortemente para esse cenário, como o convênio do Imposto sobre Circulação de Mercadorias e Serviços (ICMS) 100/97 que reduzia em 60\% a alíquota da cobrança desse imposto para os agrotóxicos e o decreto 6.006/06 que isentava alguns deles completamente de cobrança de impostos sobre produtos industrializados (IPI).

A partir da circulação de vários tipos de agrotóxicos, Londres 
(2011) esboça um fator de risco para a agricultura no Brasil, que é a comercialização de forma ampla dos agroquímicos já banidos em outros locais como Estados Unidos, China e alguns países da União Europeia, mostrando a inércia do poder público em relação a esta questão alarmante, podendo gerar efeitos negativos imensuráveis.

Essa situação se mostra preocupante na sub-bacia do rio Natuba. Ela tem um papel preponderante no abastecimento de Recife por meio do Centro de Abastecimento e Logística de Pernambuco (CEASA/PE), além de toda a região metropolitana. Rodrigues (2006), Marques et al. (2013); Nascimento (2013) e Marques (2017) relataram de forma enfática ações inadequadas verificadas como a situação do abandono das embalagens vazias de agrotóxicos de forma indiscriminada, manipulação irregular desses insumos, frequência de pulverização não condizente com as recomendações dos fabricantes.

Assim, o meio ambiente tem sido amplamente afetado, a exemplo dos recursos hídricos superficiais e subterrâneos, como revela os estudos de Nascimento (2013) no rio Natuba, quando evidenciou a presença de ingredientes ativos de agrotóxicos em amostras de água subterrânea em 18 dos 24 poços de monitoramento analisados.

Vários estudos (BRAGA; GUSMÃO; MESEL, 2006; NASCIMENTO, 2013; NORONHA; LIRA; MORAIS, 2016) relatam a existência de manejo inadequado de agrotóxicos pelos agricultores rurais da economia familiar que labutam na região drenada pela sub-bacia do rio Natuba. Essa manipulação feita de forma equivocada vem gerando diversos casos de contaminação ambiental nos compartimentos ambientais: solo, água, atmosfera e ser humano. O Brasil, bem como vários outros países, possui grande potencialidade na água subterrânea como opção à escassez da superficial. Todavia, ela vem sendo bastante degradada e apresenta um comportamento distinto do fluxo de contaminação em relação ao que acontece na superfície.

Assim, ferramentas podem ser empregadas de modo a auxiliar em um processo de gerenciamento da área, como a modelagem matemática e uso de índices aplicados a partir de fórmulas e critérios validados em estudos científicos, como Groundwater Screening
Index (GSI), Groundwater Ubiquity Score (GUS), Leaching IndeX (LIX), Leaching Index (LEACH), Relative Leaching Potential Index (RLPI) e critério do California Departament of Food and Agriculture (CDFA).

Dessa forma, o estudo objetivou calcular o potencial de lixiviação e contaminação dos princípios ativos de agrotóxicos que são utilizados na sub-bacia hidrográfica do rio Natuba.

\section{MATERIAIS E MÉTODOS}

Com o objetivo de entender a potencialidade de contaminação dos recursos hídricos subterrâneos na sub-bacia do rio Natuba foram analisados os ingredientes ativos de agrotóxicos, contidos em uma lista de 35 princípios ativos compilados por Nascimento (2013) para a região do estudo, por meio de modelagem matemática. Além desses, foram adicionados quatro outros princípios ativos (dithiocarbamatos, fentoato, malathion e spinosad) identificados nas análises laboratoriais feitas pela mesma autora nas diversas matrizes ambientais verificadas (água superficial e subterrânea, solo, alimento), perfazendo 39 princípios ativos.

\subsection{Caracterização da área em estudo}

Com cerca de $39 \mathrm{~km}^{2}$ de área de drenagem, a sub-bacia do Natuba (Figura 01) corresponde a 8,23\% da bacia do Tapacurá (SILVA, 2007; MENEZES, 2010; MENEZES et al., 2010; BARBOSA NETO et al., 2011; ARAÚJO FILHO et al., 2013; SILVA et al., 2017). A região de Natuba é dividida em: baixo, médio e alto Natuba se situando entre os municípios de Vitória de Santo Antão e Pombos - PE.

A sub-bacia do baixo Natuba está em uma área de baixa densidade de drenagem com clima tropical que favorece o intemperismo e desenvolvimento de solos profundos (SILVA et al., 2017). Ela é situada integralmente em Vitória de Santo Antão e é conhecida pela produção de hortaliças folhosas, sendo considerada por muitos como o cinturão verde do estado de Pernambuco (CAVALCANTI; FERREIRA; NASCIMENTO, 2010; RIBEIRO, 2011; NASCIMENTO, 2013). Neste sentido, Souza e Araújo (2011) comentam que essa região produz e fornece cerca de $60 \%$ e hortaliças folhosas do que é consumido na região metropolitana do Recife. 
Figura 01 - Caracterização da sub-bacia hidrográfica do Natuba, Vitória de Santo Antão - PE

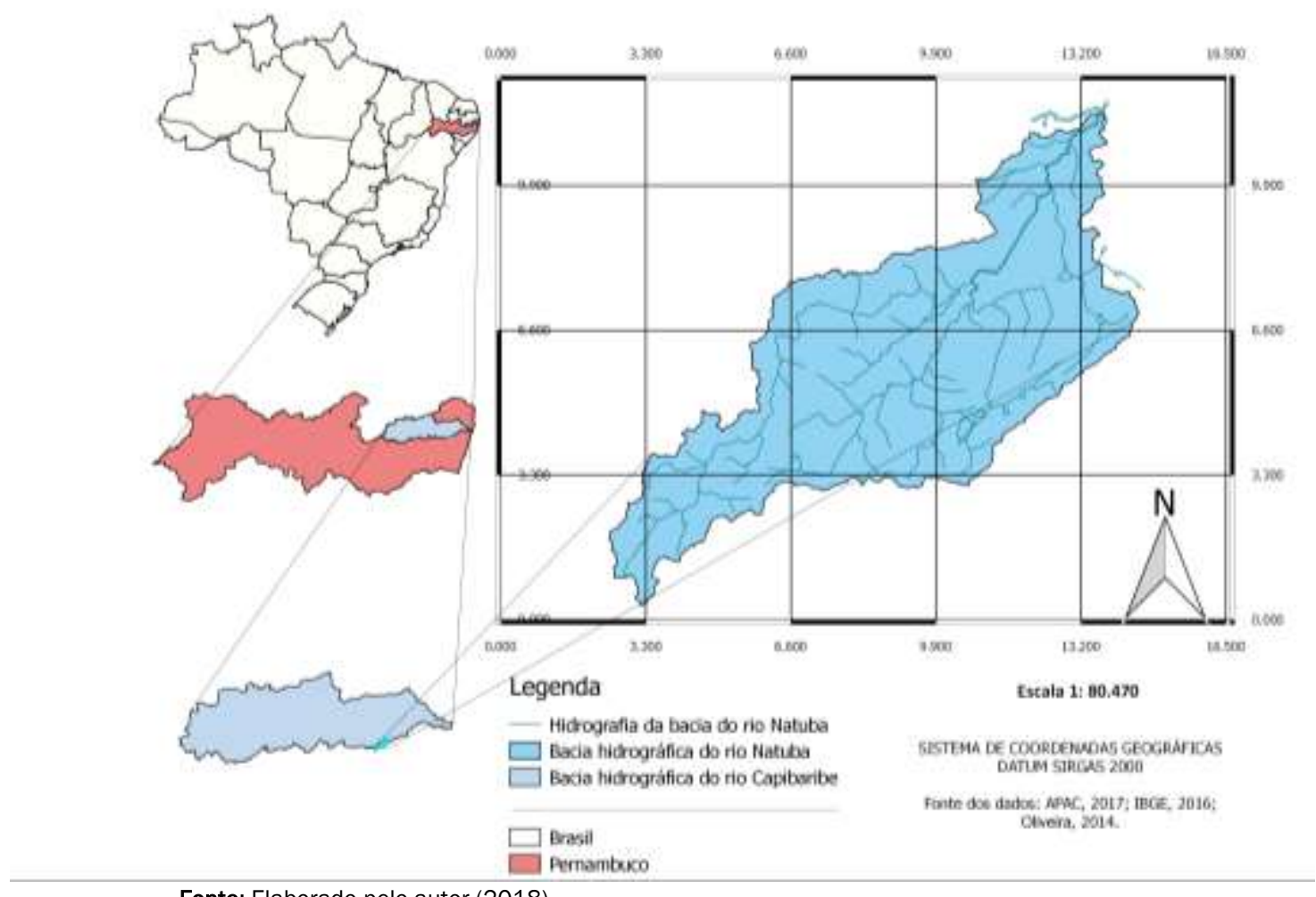

Fonte: Elaborado pelo autor (2018)

\section{2. Índices de potencial de lixiviação selecionados}

Para entender a potencialidade de contaminação dos princípios ativos de agrotóxicos utilizados na região, utilizaram-se alguns índices de potencial de lixiviação validados e reconhecidos na literatura científica.

\subsubsection{Critério CDFA}

O California Departament of Food and Agriculture (WILKERSON; KIM, 1986) criou critérios para classificar os agrotóxicos conforme o potencial de contaminar os recursos hídricos subterrâneos. Para tal divisão, foram propostas duas cláusulas, a saber:

a) o valor de coeficiente de adsorção ao carbono orgânico $\left(\mathrm{k}_{\text {oc }}\right)$ deve ser menor que $512 \mathrm{~mL} / \mathrm{g}$ ou solubilidade em água maior que $7 \mathrm{mg} / \mathrm{L}$; e

b) meia vida de hidrólise maior que 13 dias ou meia vida de degradação no solo maior que 11 dias.

Caso determinado agrotóxico atenda aos dois critérios, o CDFA considera este um produto químico com grande capacidade de degradação ambiental. 0 koc expressa a capacidade do princípio ativo permanecer adsorvido à superfície do solo, a solubilidade em água representa a aptidão a ser dissolvido em água, a meia vida de hidrólise e de degradação no solo apresentam o tempo em dias necessário para que aquela substância seja degradada à metade.

\subsection{2. Índice GSI}

O GSI, desenvolvido por Bishop (1986), utiliza os valores de solubilidade $(\mathrm{mg} / \mathrm{L})$, tempo de meia vida no solo $\mathrm{T}^{112}$ (dias) além do coeficiente de partição octanol-água $\mathrm{K}_{\mathrm{ow}}(\mathrm{mL} / \mathrm{g})$ por meio da Fórmula 01. Ele traz um parâmetro distinto dos demais: coeficiente
Kow que se trata de afinidade da substância com as fases polar e apolar.

$$
G S I=\ln \left[\left(S . T^{1 \backslash 2}\right) / \log K_{o w}\right.
$$

Este índice é bastante útil para distinguir as substâncias que terão tendência para contaminar as águas subterrâneas (OLIVEIRA et al., 2016a). Os valores informados podem ser agrupados conforme as faixas descritas por Oliveira et al. (2016b):

GSI<1: Contaminação improvável (Cl)

$1<\mathrm{GSI}$ < 3: possível contaminação (PC)

GSI > 3: contaminação provável (CP)

GSI > 5: contaminante deve ser tratado com atenção especial (CE)

\subsection{3. Índice de $L E A C H$}

O índice de LEACH (LASKOWSKI et al., 1982) assinala a potencialidade de contaminação tanto de lençóis subterrâneos quanto dos superficiais (ARMAS et al., 2005). Todavia, para este estudo, será considerado somente o recurso hídrico subterrâneo.

$$
\mathrm{LEACH}=\left[\left(\mathrm{Ws} . \mathrm{T}^{1} \backslash 2 \mathrm{solo}\right) /\left(\mathrm{Vp} . \mathrm{K}_{\mathrm{oc}}\right)\right]
$$

Sendo Ws a solubilidade em água $(\mathrm{mg} / \mathrm{L}), \mathrm{T}^{1} \backslash 2$ o tempo de meia vida no solo (dias) solo, Vp o vapor de pressão (MPa) e Koc o coeficiente de adsorção ao carbono orgânico (BRITTO, 2011).

Segundo Britto (2011), dentre essas variáveis, a solubilidade tem um peso muito grande no resultado e, consequentemente, na potencialidade de contaminação dos recursos hídricos subterrâneos tendo em vista representar a quantidade máxima 
desse produto que pode dissolver em uma quantidade padrão de água.

Armas et al. (2005) comentam que o índice LEACH, ao contrário da maioria, não enquadra os princípios ativos em classes de potencialidade de contaminação ambiental, mas o seu entendimento deve partir do raciocínio de que quanto maior for o valor, maior será a tendência de lixiviação do produto.

\subsection{4. Índice de LIX}

O índice de LIX foi desenvolvido para fornecer uma noção clara sobre a potencialidade de lixiviação de alguns tipos de agrotóxicos em uma escala que varia de 0 a 1 . Ele se mostra como uma meIhor ferramenta se comparado ao índice de GUS, tendo em vista que este último propõe valores negativos que dificultam a interpretação. A ideia principal do índice é de ratificar quais produtos devem sofrer uma análise posterior sobre a sua potencial característica lixiviadora (SPADOTTO, 2002).

A fórmula estabelecida por Spadotto (2002) é:

$$
L I X=\exp \left(-k \cdot K_{o c}\right)
$$

Em que k é a taxa constante de primeira ordem do pesticida e koc 0 coeficiente de adsorção ao carbono orgânico.

\subsubsection{GUS}

Proposto por Gustafson em 1989, o índice de GUS faz uso do coeficiente de adsorção ao carbono orgânico $\left(\mathrm{K}_{\mathrm{oc}}\right)$ e o tempo de meia vida do agrotóxico no solo $\left(T^{1} \backslash 2\right)$. Foi criado de forma a ser uma ferramenta que trabalha somente com dois dados físicoquímicos, dispensando outros frequentemente utilizados como a solubilidade em água, meia vida de hidrólise e constante de Henry.

A fórmula utilizada por Gustafson (1989) é a seguinte:

$$
\text { GUS }=\log \left(T^{\top 1 / 2}\right) *\left(4-\log K_{o c}\right)
$$

\subsection{6. $R L P I$}

Hornsby, Buttler e Brown (1993), por sua vez, criaram um índice relativo, ou seja, necessita da presença de outros agrotóxicos para permitir uma comparação entre eles. Segundo os autores, quanto menor o valor, maior o potencial de lixiviação.

$$
\mathrm{RLPI}=10 *\left(\mathrm{~K}_{\mathrm{oc}} / \mathrm{T}_{1 / 2}\right)
$$

\subsection{Comparação entre os índices}

Inicialmente, com o intuito de comparar as estimativas de lixiviação de agrotóxicos utilizados na sub-bacia, escolheram-se seis índices caracterizados por serem de simples aplicação visando evidenciar o potencial de lixiviação dos princípios ativos utilizados na região, de modo a verificar a concordância de resultados para um mesmo princípio ativo. Essa prática tende a diminuir as lacunas existentes nos índices, segundo Souza e Green (2016), como a necessidade de confirmação de cenários.

Os índices GSI, GUS, LIX, LEACH e RLPI, a exceção do critério de CFDA, pelo fato de não possuir output numérico, foram comparados por meio de análise das variâncias ( $p$-valor 0,05$)$ com o intuito de saber se eles tendem a dar resultados semelhantes para que os tomadores de decisão possam escolher quais índices utilizar, de forma associada ou não, além de permitir a percepção das deficiências, tendo em vista a simplicidade envolvida. A base de dados considerada apresentou distribuição normal segundo o teste de Kolmorov-Smirnov (LOPES; CASTELO BRANCO; SOARES, 2013). Para isso, foi realizada a padronização dos dados em relação à média para que os dados pudessem estar na mesma faixa de valores, tendo em vista que cada índice possui resultados em sua própria escala, o que inviabilizaria a comparação.

A maioria dos elementos expressos foram coletados na base de dados Pesticide Properties DataBase (PPDB) desenvolvido pela Universidade de Hertfordshire, na unidade de Agriculture and Environment Research Unit (AERU) da Inglaterra (OLIVEIRA et al., 2016b). A base de dados possui informações de cerca de 2.300 substâncias, além de mais de 700 metabólitos com parâmetros sobre saúde humana, qualidade ambiental e riscos a biodiversidade (LEWIS et al., 2016).

\section{RESULTADOS E DISCUSSÃO}

Após os cálculos, foram obtidos os índices (Tabela 01).

Ao se analisar os agrotóxicos pelo critério da CDFA, embora não haja nenhum tipo de agrupamento específico para os ingredientes nesse critério que tenham atendido a todos os quatro parâmetros, é importante registrar que 2,4 D sal dimetilamina, carbofurano, hexazinone, imidacloprido e thiamethoxam tiveram esse comportamento e, sendo assim, necessitam de uma maior atenção na hora da escolha do produto a ser aplicado.

Ao se avaliar esses princípios ativos pelo GSI, destacaram-se os seguintes agrotóxicos dentre os demais (até mesmo dentro da classe de contaminantes que devem ser tratado de forma especial): paraquate, dicloreto de paraquate, 2,4 D sal dimetilamina, etefom, methomyl, hexazinone, thiamethoxam, cartape, 2,4 D, imidacloprido, methalaxyl M, picloram e glifosato. No total, $56,41 \%$ entraram nas classes onde o contaminante deve ser tratado com atenção especial (CE) ou na classe de contaminação provável (CP).

Quanto ao índice de GUS, houve uma taxa de 28,20\% de agrotóxicos registrados como lixiviadores ou em transição. Corroborando com o resultado, Nascimento (2013), ao analisar os seus dados para a mesma área de estudo, encontrou um percentual de $28,94 \%$ para esse mesmo índice.

As diferenças apresentadas pelos índices de GUS e GSI podem ser entendidas, segundo Oliveira et al. (2016a, 2016b), pelo fato de que o segundo utiliza o coeficiente de partição entre água/octanol e o primeiro faz uso do tempo de meia vida do agrotóxico no solo e do $\mathrm{K}_{\mathrm{oc}}$.

Pfeiffer (2010), quando também aplicou o GUS, obteve o mesmo resultado para os ingredientes ativos trabalhados que coincidiram com esse estudo como, dentre outros, a azoxistrobina (transição), deltametrina (não lixivia), imidacloprido (lixivia) e diuron (transição).

O índice de LIX, por sua vez, teve 20,51\% dos agrotóxicos nas classes de transição ou como lixiviadores. Esse fato se aproxima 
do resultado do índice de GUS, índices divididos em não lixiviadores, transição e lixiviadores. Todavia, esses valores diferem dos percentuais de CDFA, no que tange ao quantitativo de p.a. potencialmente contaminantes, provavelmente pelo fato de não ser alocado em faixas de potencialidade como os primeiros

Tabela 01 - Resultados dos índices: LIX, CDFA, GSI, LEACH, GUS e RLPI

\begin{tabular}{|c|c|c|c|c|c|c|}
\hline Ingredientes ativos & CDFA & GSI & GUS & LEACH $\left(\log _{10}\right)$ & LIX & RLPI \\
\hline $2,4 \mathrm{D}$ & $\mathrm{PC}$ & 11,77 (CE) & $1,55(\mathrm{NL})$ & 5,48 & $0,00(\mathrm{NL})$ & 89,3 \\
\hline 2,4 D sal dimetilamina & $\mathrm{PC}$ & 17,09 (CE) & $3,58(\mathrm{~L})$ & 16,97 & $0,39(\mathrm{~L})$ & 13,5 \\
\hline Abamectina & - PC & $2,06(\mathrm{PC})$ & $0,36(\mathrm{NL})$ & 0,22 & $0,00(\mathrm{NL})$ & 1964,4 \\
\hline Alfacipermetrina & $-P C$ & $-3,67(\mathrm{Cl})$ & $-1,18(\mathrm{NL})$ & $-2,14$ & $0,00(\mathrm{NL})$ & 16539,7 \\
\hline Azoxistrobina & - PC & 5,34 (CE) & $2,33(\mathrm{~T})$ & 6,90 & $0,01(\mathrm{~T})$ & 75,5 \\
\hline Beta ciflutrina & $-P C$ & $-5,93(\mathrm{Cl})$ & $-0,90(\mathrm{NL})$ & $-2,36$ & $0,00(\mathrm{NL})$ & 49461,5 \\
\hline Carbofurano & PC & 8,55 (CE) & 3,81 (L) & 3,66 & $0,55(\mathrm{~L})$ & 8,6 \\
\hline Cartape & Inconclusivo* & 13,35 (CE) & $1,24(\mathrm{NL})$ & 14,34 & $0,00(\mathrm{NL})$ & 82,5 \\
\hline Cipermetrina & $-P C$ & $-2,28(\mathrm{Cl})$ & $-2,12(\mathrm{NL})$ & $-1,82$ & $0,00(\mathrm{NL})$ & 26041,6 \\
\hline Clorantraniliprole & PC & 5,21 (CE) & $4,00(\mathrm{~L})$ & 8,36 & $0,66(\mathrm{~L})$ & 6,0 \\
\hline Deltametrina & $-P C$ & $-7,47(\mathrm{Cl})$ & $-3,35(\mathrm{NL})$ & $-4,68$ & $0,00(\mathrm{NL})$ & 787692,3 \\
\hline Dicloreto de paraquate & PC & 17,73 (CE) & $-2,56(\mathrm{NL})$ & 5,35 & $0,00(\mathrm{NL})$ & 2739,7 \\
\hline Difenoconazole & PC & $6,10(\mathrm{CE})$ & $0,45(\mathrm{NL})$ & 3,98 & $0,00(\mathrm{NL})$ & 470,7 \\
\hline Dithiocarbamatos & - & - & - & - & - & - \\
\hline Diuron & PC & 6,84 (CE) & $2,05(\mathrm{~T})$ & 3,45 & $0,00(\mathrm{NL})$ & 107,6 \\
\hline Espiromesifeno & - PC & $-2,14(\mathrm{Cl})$ & $-0,30(\mathrm{NL})$ & $-2,60$ & $0,00(\mathrm{NL})$ & 75365,8 \\
\hline Etefom & $\mathrm{PC}$ & 15,95 (CE) & $0,72(\mathrm{NL})$ & 3,79 & $0,00(\mathrm{NL})$ & 1587,5 \\
\hline Famaxadone & $-P C$ & $-0,65(\mathrm{Cl})$ & $0,67(\mathrm{NL})$ & $-0,00$ & $0,00(\mathrm{NL})$ & 936,0 \\
\hline Fenoxaprope-p-etílico & $-P C$ & $-2,79(\mathrm{Cl})$ & $0,02(\mathrm{NL})$ & $-1,33$ & $0,00(\mathrm{NL})$ & 283850,0 \\
\hline Fentoato & PC & 4,64 (CP) & $1,54(\mathrm{NL})$ & $-1,13$ & $0,00(\mathrm{NL})$ & 285,7 \\
\hline Fluazifop-P-butil & $-P C$ & $-1,57(\mathrm{Cl})$ & $0,00(\mathrm{NL})$ & $-2,64$ & $0,00(\mathrm{NL})$ & 33940,0 \\
\hline Glifosato & PC & 10,80 (CE) & $1,00(\mathrm{NL})$ & 3,92 & $0,00(\mathrm{NL})$ & 949,3 \\
\hline Glifosato potássio & Inconclusivo* & - & $-1,67(\mathrm{NL})$ & - & $0,00(\mathrm{NL})$ & 7208,3 \\
\hline Hexazinone & PC & $14,90(\mathrm{CE})$ & $4,58(\mathrm{~L})$ & 6,33 & $0,70(\mathrm{~L})$ & 5,1 \\
\hline Imidacloprido & PC & 12,22 (CE) & 3,61 (L) & 9,03 & $0,39(\mathrm{~L})$ & 13,7 \\
\hline Lambda-cialotrina & $-P C$ & $-1,83(\mathrm{Cl})$ & $-3,26(N L)$ & $-1,81$ & $0,00(\mathrm{NL})$ & 16211,8 \\
\hline Linurom & $P C$ & 7,11 (CE) & $1,89(\mathrm{~T})$ & 0,93 & $0,00(\mathrm{NL})$ & 146,3 \\
\hline Malathion & - PC & $2,21(P C)$ & - 0,57 (NL) & $-2,34$ & $0,00(\mathrm{NL})$ & 105882,3 \\
\hline Mancozebe & - PC & $-0,76(\mathrm{Cl})$ & $-1(\mathrm{NL})$ & $-1,32$ & $0,00(\mathrm{NL})$ & 99800,0 \\
\hline Metalaxyl M & PC & 11,50 (CE) & $1,45(\mathrm{NL})$ & 2,49 & $0,00(\mathrm{NL})$ & 250,7 \\
\hline Methomyl & $P C$ & 15,26 (CE) & $1,81(\mathrm{~T})$ & 3,87 & $0,00(\mathrm{NL})$ & 102,8 \\
\hline Nonil fenol etoxilado & Inconclusivo* & - & - & - & - & - \\
\hline Paraquate & $\mathrm{PC}$ & 19,83 (CE) & $-6,95(\mathrm{NL})$ & 5,26 & $0,00(\mathrm{NL})$ & 3333,3 \\
\hline Pencycurom & $-\mathrm{PC}$ & 1,66 (PC) & 0,47 (NL) & 1,02 & $0,00(\mathrm{NL})$ & 687,7 \\
\hline Spinosad & $\mathrm{PC}$ & 6,71 (CE) & - 0,62 (NL) & 3,65 & $0,00(\mathrm{NL})$ & 24714,2 \\
\hline Picloram & PC & 10,09 (CE) & $5,54(\mathrm{~L})$ & 7,64 & $0,90(\mathrm{~L})$ & 1,5 \\
\hline Tebuconazole & PC & 6,41 (CE) & $1,80(\mathrm{NL})$ & 3,24 & $0,00(\mathrm{NL})$ & 158,7 \\
\hline Thiamethoxam & PC & 14,26 (CE) & $3,82(L)$ & 8,74 & $0,46(\mathrm{~L})$ & 11,2 \\
\hline Trifloxixtrobina & $-P C$ & $-3,07(\mathrm{Cl})$ & $-0,29(\mathrm{NL})$ & $-1,59$ & $0,00(\mathrm{NL})$ & 69911,7 \\
\hline
\end{tabular}

Legenda: Cl- contaminação improvável; PC- possível contaminação; -PC- não há potencialidade de contaminação; CP- contaminação provável; CE- contaminante que deve ser tratado com especial consideração; L- lixivia; NL- não lixivia; T- transição; *Inconclusivo devido à falta de dados que compromete a aplicação dos requisitos propostos pelos autores.

Segundo o índice, clorantraniliprole, 2,4 D sal dimetilamina, carbofurano, hexazinone, imidacloprido, picloram e thiamethoxam são compostos lixiviadores e azoxistrobina se encontra na faixa de transição.

No índice LEACH, os valores apresentados foram expressos em escala logarítmica visando uma melhor comparação com índice de GUS (ARMAS et al., 2005), ou seja, para fins de avaliação e estudos foi considerado somente o logaritmo de base 10. Nesse sentido, destacaram-se pelo alto índice de lixiviação, respectivamente: 2,4 D sal dimetilamina, cartape, imidacloprido, thiamethoxam, clorantraniliprole, picloram, azoxistrobina, hexazinone, 2,4 D, dicloreto de paraquate e paraquate.

Armas et al. (2005) avaliaram por meio do índice de GUS e LEACH alguns princípios ativos de agrotóxicos utilizados na produção de cana-de-açúcar perto da calha do rio Corumbataí localizado no estado de São Paulo. Segundo os autores, esse passo é essencial para indicar quais os agrotóxicos são essenciais em um programa de monitoramento por parte do governo.

Com relação ao RLPI, quanto menor o valor, maior é a capacidade de lixiviar (NASCIMENTO, 2013), ao contrário do LEACH. Os agrotóxicos propensos a lixiviar, segundo RLPI, que mais se destacaram foram: picloram, hexazinone, clorantraniliprole, carbofurano, thiamethoxam, 2,4 D sal dimetilamina, imidacloprido, azoxistrobina, cartape e 2,4 D.

Assim, pelo fato de os índices LEACH e RLPI não enquadrarem os ingredientes ativos em classes quanto ao potencial de contaminação ambiental, estimou-se que, para fins de avaliação, a média aritmética simples entre os valores de cada um dos dois seria o divisor para definir os que seriam possíveis contaminantes e os que não seriam classificados como tal. Dessa forma, $48,71 \%$ e $89,74 \%$ foram os percentuais de ingredientes alocados como potencialmente contaminadores para LEACH e RLPI, respectivamente. 
Soares, Faria e Rosa (2017) inventariaram os principais ingredientes ativos no município de Campo Novo do Parecis - Mato Grosso e, posteriormente, analisaram as suas propriedades físicoquímicas alocando-as no índice de GUS e pelo critério da United States Environmental Agency (USEPA). A partir desse estudo, concluiu-se que $26,1 \%$ dos ingredientes ativos tem potencial de contaminação de lençóis subterrâneos.

Com o uso desses índices, Oliveira et al. (2016a) analisaram os agrotóxicos aprovados para a cultura do tomate por meio do índice de GUS que mostrou que $70 \%$ dos compostos não tem o risco de sofrer lixiviação e os 30\% restantes estão na faixa de transição. Já com relação ao GSI, 41,7\% devem receber tratamento especial, $20,8 \%$ não tem possibilidade de contaminação e 37,2\% existe uma provável contaminação. As diferenças entre os índices podem ser devido ao índice GSI utilizar o Kow e solubilidade, ao contrário do GUS. Eles oferecem a afinidade das fases polar e apolar, além da capacidade de solubilizar em água a uma dada temperatura, respectivamente.

Por sua vez, Rodríguez (2013) analisou água subterrânea na área de Santa Isabel em Porto Rico e estudou a potencialidade de lixiviação dos pesticidas encontrados, por meio do índice de GUS, e concluiu que $72,72 \%$ dos princípios ativos foram indicados como lixiviadores ou em transição, mostrando a aplicabilidade do índice.

Frente ao exposto, por meio do estudo de Oliveira et al. (2016b), é possível perceber que os métodos de quantificação executados a partir de análises laboratoriais de contaminação por agrotóxicos são muito caros. Sendo assim, uma estimativa inicial dos princípios ativos por meio de uma análise de informações já disponíveis em bancos de dados é bastante viável.

Os seguintes princípios ativos apareceram em todos os índices analisados apontando de modo mais enfático a potencialidade de contaminar o meio ambiente: 2,4 D; 2,4 D sal dimetilamina; carbofurano; clorantraniliprole; hexazinone; imidacloprido; picloram, thiamethoxam e methomyl.

A Tabela 02 expressa a hierarquização entre todos os agrotóxicos selecionados e utilizados na sub-bacia. O critério CDFA não foi considerado para essa fase do estudo tendo em vista não trabalhar com classes numéricas.

Tabela 02 - Hierarquização entre os princípios ativos analisados quanto à potencialidade de contaminação

\begin{tabular}{|c|c|c|c|c|c|c|}
\hline Ingredientes ativos & GSI & GUS & LEACH $\left(\log _{10}\right)$ & LIX & RLPI & Classificação geral \\
\hline $\begin{array}{c}\text { Picloram } \\
\text { Hexazinone }\end{array}$ & $\begin{array}{l}13^{\circ} \\
06^{\circ}\end{array}$ & $\begin{array}{l}01^{\circ} \\
02^{\circ}\end{array}$ & $\begin{array}{l}06^{\circ} \\
08^{\circ}\end{array}$ & $\begin{array}{l}01^{\circ} \\
02^{\circ}\end{array}$ & $\begin{array}{l}01^{\circ} \\
02^{\circ}\end{array}$ & $\begin{array}{l}1 \\
2\end{array}$ \\
\hline $\begin{array}{l}\text { Clorantraniliprole } \\
\text { Carbofurano }\end{array}$ & $\begin{array}{l}21^{\circ} \\
14^{\mathrm{a}}\end{array}$ & $\begin{array}{l}03^{\circ} \\
05^{\circ}\end{array}$ & $\begin{array}{l}05^{\circ} \\
16^{\circ}\end{array}$ & $\begin{array}{l}03^{\circ} \\
04^{\circ}\end{array}$ & $\begin{array}{l}03^{\circ} \\
04^{\circ}\end{array}$ & $\begin{array}{l}3 \\
4\end{array}$ \\
\hline $\begin{array}{l}\text { Thiamethoxam } \\
\text { 2,4 D sal dimetilamina } \\
\text { Imidacloprido }\end{array}$ & $\begin{array}{l}07^{\circ} \\
03^{\circ} \\
09^{\circ}\end{array}$ & $\begin{array}{l}04^{\circ} \\
07^{\circ} \\
06^{\circ}\end{array}$ & $\begin{array}{l}04^{\circ} \\
01^{\circ} \\
03^{\circ}\end{array}$ & $\begin{array}{l}05^{\circ} \\
06^{\circ} \\
06^{\circ}\end{array}$ & $\begin{array}{l}05^{\circ} \\
06^{\circ} \\
07^{\circ}\end{array}$ & $\begin{array}{l}4 \\
6 \\
6\end{array}$ \\
\hline Azoxistrobina & $20^{\circ}$ & $08^{\circ}$ & $07^{\circ}$ & $07^{\circ}$ & $08^{\circ}$ & 7 \\
\hline Cartape & $08^{\circ}$ & $16^{\circ}$ & $02^{\circ}$ & $08^{\circ}$ & $09^{\circ}$ & 8 \\
\hline Dicloreto de paraquate & $02^{\circ}$ & $34^{\circ}$ & $10^{\circ}$ & $08^{\circ}$ & $23^{\circ}$ & 9 \\
\hline Diuron & $16^{\circ}$ & $09^{\circ}$ & $18^{\circ}$ & $08^{\circ}$ & $12^{\circ}$ & 9 \\
\hline $\begin{array}{c}2,4 \mathrm{D} \\
\text { Paraquat }\end{array}$ & $\begin{array}{l}10^{\circ} \\
01^{\circ}\end{array}$ & $\begin{array}{l}13^{\circ} \\
37^{\circ}\end{array}$ & $\begin{array}{l}09^{\circ} \\
11^{\circ}\end{array}$ & $\begin{array}{l}08^{\circ} \\
08^{\circ}\end{array}$ & $\begin{array}{l}10^{\circ} \\
24^{\circ}\end{array}$ & $\begin{array}{l}10 \\
10\end{array}$ \\
\hline $\begin{array}{l}\text { Methomyl } \\
\text { Glifosato }\end{array}$ & $\begin{array}{l}05^{\circ} \\
12^{\circ}\end{array}$ & $\begin{array}{l}11^{\circ} \\
17^{\circ}\end{array}$ & $\begin{array}{l}14^{\circ} \\
13^{\circ}\end{array}$ & $\begin{array}{l}08^{\circ} \\
08^{\circ}\end{array}$ & $\begin{array}{l}11^{\circ} \\
20^{\circ}\end{array}$ & $\begin{array}{l}11 \\
13\end{array}$ \\
\hline $\begin{array}{l}\text { Tebuconazole } \\
\text { Linurom }\end{array}$ & $\begin{array}{l}18^{\circ} \\
15^{\circ}\end{array}$ & $\begin{array}{l}12^{\circ} \\
10^{\circ}\end{array}$ & $\begin{array}{l}19^{\circ} \\
22^{\circ}\end{array}$ & $\begin{array}{l}08^{\circ} \\
08^{\circ}\end{array}$ & $\begin{array}{l}14^{\circ} \\
13^{\circ}\end{array}$ & $\begin{array}{l}13 \\
14\end{array}$ \\
\hline Fentoato & $22^{\circ}$ & $14^{\circ}$ & $25^{\circ}$ & $08^{\circ}$ & $16^{\circ}$ & 15 \\
\hline $\begin{array}{c}\text { Metalaxyl M } \\
\text { Etefom } \\
\text { Spinosad } \\
\text { Difenoconazole } \\
\text { Famaxadone }\end{array}$ & $\begin{array}{l}11^{\circ} \\
04^{\circ} \\
17^{\circ} \\
19^{\circ} \\
26^{\circ}\end{array}$ & $\begin{array}{l}15^{\circ} \\
18^{\circ} \\
28^{\circ} \\
21^{\circ} \\
19^{\circ}\end{array}$ & $\begin{array}{l}20^{\circ} \\
15^{\circ} \\
17^{\circ} \\
12^{\circ} \\
24^{\circ}\end{array}$ & $\begin{array}{l}08^{\circ} \\
08^{\circ} \\
08^{\circ} \\
08^{\circ} \\
08^{\circ}\end{array}$ & $\begin{array}{l}15^{\circ} \\
21^{\circ} \\
28^{\circ} \\
17^{\circ} \\
19^{\circ}\end{array}$ & $\begin{array}{l}15 \\
17 \\
17 \\
18 \\
19\end{array}$ \\
\hline $\begin{array}{l}\text { Pencycurom } \\
\text { Abamectina }\end{array}$ & $\begin{array}{l}25^{\circ} \\
24^{\circ}\end{array}$ & $\begin{array}{l}20^{\circ} \\
22^{\circ}\end{array}$ & $\begin{array}{l}21^{\circ} \\
23^{\circ}\end{array}$ & $\begin{array}{l}08^{\circ} \\
08^{\circ}\end{array}$ & $\begin{array}{l}18^{\circ} \\
22^{\circ}\end{array}$ & $\begin{array}{l}20 \\
21\end{array}$ \\
\hline Fenoxaprope-p-etílico & $32^{\circ}$ & $23^{\circ}$ & $27^{\circ}$ & $08^{\circ}$ & $36^{\circ}$ & 25 \\
\hline Mancozebe & $27^{\circ}$ & $30^{\circ}$ & $26^{\circ}$ & $08^{\circ}$ & $34^{\circ}$ & 27 \\
\hline Lambda-cialotrina & $29^{\circ}$ & $35^{\circ}$ & $29^{\circ}$ & $08^{\circ}$ & $26^{\circ}$ & 29 \\
\hline Fluazifop-P-butil & $28^{\circ}$ & $24^{\circ}$ & $35^{\circ}$ & $08^{\circ}$ & $30^{\circ}$ & 29 \\
\hline Glifosato potássio & - & $32^{\circ}$ & - & $08^{\circ}$ & $25^{\circ}$ & 29 \\
\hline Beta ciflutrina & $35^{\circ}$ & $29^{\circ}$ & $33^{\circ}$ & $08^{\circ}$ & $31^{\circ}$ & 30 \\
\hline Cipermetrina & $31^{\circ}$ & $33^{\circ}$ & $30^{\circ}$ & $08^{\circ}$ & $29^{\circ}$ & 30 \\
\hline Alfacipermetrina & $34^{\circ}$ & $31^{\circ}$ & $31^{\circ}$ & $08^{\circ}$ & $27^{\circ}$ & 31 \\
\hline Trifloxixtrobina & $33^{\circ}$ & $25^{\circ}$ & $28^{\circ}$ & $08^{\circ}$ & $32^{\circ}$ & 33 \\
\hline Espiromesifeno & $30^{\circ}$ & $26^{\circ}$ & $34^{\circ}$ & $08^{\circ}$ & $33^{\circ}$ & 34 \\
\hline Malathion & $23^{\circ}$ & $27^{\circ}$ & $32^{\circ}$ & $08^{\circ}$ & $35^{\circ}$ & 34 \\
\hline Deltametrina & $36^{\circ}$ & $36^{\circ}$ & $36^{\circ}$ & $08^{\circ}$ & $37^{\circ}$ & 36 \\
\hline Dithiocarbamatos & - & - & - & - & - & - \\
\hline Nonil fenol etoxilado & - & - & - & - & - & - \\
\hline
\end{tabular}


A partir da hierarquização dos princípios ativos, para os cinco índices de output numérico, analisou-se as posições no ranking para cada ingrediente. Para tanto, foi escolhido o conjunto das duas classificações (dentre as cinco) mais próximas entre si, e calculou-se a média aritmética simples para estabelecer a classificação geral, com o intuito de possibilitar a escolha, caso haja a necessidade de aplicação, do princípio ativo com um indicativo de menor potencial de risco para o meio ambiente.

Quando houve dois pares de valores com a mesma proximidade, escolheu-se o par com valor mais próximo de 1 . Para fins de exemplificação, o thiamethoxam obteve as seguintes posições nos rankings dos índices: $7^{\circ}$ (GSI), $4^{\circ}$ (GUS), $4^{\circ}$ (LEACH), $5^{\circ}$ (LIX) e $5^{\circ}$ (RLPI). Então, há dois pares que poderia se trabalhar: $\left(4^{\circ}, 4^{\circ}\right)$ e $\left(5^{\circ}\right.$, $\left.5^{\circ}\right)$, mas escolheu-se o par $\left(4^{\circ}, 4^{\circ}\right)$ por ter mais proximidade com 1 . Efetuou-se, assim, a média entre as duas que resultou na posição geral $4^{\circ}$. Assim, picloram, hexazinone, clorantraniliprole, carbofurano, thiamethoxam, 2,4 D Sal dimetilamina, imidacloprido, azoxistrobina, cartape, dicloreto de paraquate, diuron, 2,4 D, paraquate, methomyl, glifosato, tebuconazole, linurom, fentoato, methalaxyl M estiveram alocados nas 15 primeiras colocações, indicando que são os agrotóxicos com maior potencial de risco ao meio ambiente.

Dentre os cinco índices utilizados, GSI, GUS e LIX são os mais indicados para programas de gerenciamento, por serem classificados em classes de potencial lixiviação, facilitando a tomada de decisão, ao contrário do RLPI e LEACH que são aplicados simplesmente para comparar os resultados dos índices entre os ingredientes ativos.

Porém, analisando-se especificamente os compostos azoxistrobina, imidacloprido, tebuconazole e fentoato, o imidacloprido, verificou-se que estes obtiveram destaque na potencialidade de lixiviação, fato confirmado no estudo de Saatman (2016), em que o imidacloprido foi modelado utilizando o software ARAquá. Por sua vez, Nascimento (2013) encontrou esses princípios ativos nos recursos hídricos subterrâneos da subbacia do Natuba, a saber: azoxistrobina na água subterrânea, superficial, solo e hortaliças; o imidacloprido foi observado nas matrizes água subterrânea, solo e hortaliças; o tebuconazole na água subterrânea, superficial, hortaliças; e fentoato na água subterrânea. Esses resultados corroboram com os índices apresentados nesse trabalho e fornecem base para simulação de cenários futuros de possível contaminação ambiental na região em estudo.

\subsection{Análise dos índices}

Cada índice possui uma variabilidade de dados específica em um intervalo numérico previamente definido. O GSI, GUS e LEACH assumem a possibilidade de valores negativos e indicam que, quanto maior o número, maior a potencialidade do ingrediente lixiviar. Já o RLPI evidencia que, quanto menor o seu valor, maior é a possibilidade de percolar no solo. O LIX, por sua vez, está contido no intervalo entre 0 e 1 (sem possibilidade de números negativos).

Para fins de uma melhor verificação desse grau de interligação entre os índices que possuem variáveis numéricas, excluindo-se o critério CDFA, padronizou-se todos os dados para a mesma unidade de medida (Tabela 03). A seguir, verificou-se que, apesar da heterogeneidade existente entre os índices, devido aos seus intervalos específicos, não há influência na interpretação sobre a potencialidade de lixiviar dos agrotóxicos (Tabela 04). A maioria deles utilizam dados de entrada semelhantes justificando que, mesmo tendo aparentemente valores discrepantes, eles se comportam de uma forma semelhante na compreensão da realidade simulada. 
Tabela 03 - Valores de GSI, GUS, LEACH, LIX e RLPI padronizados

\begin{tabular}{|c|c|c|c|c|c|}
\hline Ingredientes ativos & GSIp & GUSp & LEACHp & LIXp & RLPIp \\
\hline $\begin{array}{c}2,4 \mathrm{D} \\
2,4 \mathrm{D} \text { sal dimetilamina }\end{array}$ & $\begin{array}{l}0,968 \\
1,858\end{array}$ & $\begin{array}{l}1,217 \\
4,122\end{array}$ & $\begin{array}{l}0,916 \\
4,934\end{array}$ & $\begin{array}{l}-0,850 \\
2,083\end{array}$ & $\begin{array}{l}-1,000 \\
-1,000\end{array}$ \\
\hline $\begin{array}{c}\text { Abamectina } \\
\text { Alfacipermetrina }\end{array}$ & $\begin{array}{l}-0,656 \\
-1,614\end{array}$ & $\begin{array}{l}-0,485 \\
-2,688\end{array}$ & $\begin{array}{l}-0,923 \\
-1,748\end{array}$ & $\begin{array}{l}-0,850 \\
-0,850\end{array}$ & $\begin{array}{l}-0,992 \\
-0,932\end{array}$ \\
\hline Azoxistrobina & $-0,107$ & 2,333 & 1,413 & $-0,774$ & $-1,000$ \\
\hline Beta ciflutrina & $-1,992$ & $-2,288$ & $-1,825$ & $-0,850$ & $-0,795$ \\
\hline Carbofurano & 0,430 & 4,451 & 0,280 & 3,286 & $-1,000$ \\
\hline Cartape & 1,232 & 0,774 & 4,014 & $-0,850$ & $-1,000$ \\
\hline Cipermetrina & $-1,381$ & $-4,033$ & $-1,636$ & $-0,850$ & $-0,892$ \\
\hline Clorantraniliprole & $-0,129$ & 4,722 & 1,923 & 4,113 & $-1,000$ \\
\hline Deltametrina & $-2,249$ & $-5,793$ & $-2,636$ & $-0,850$ & 2,262 \\
\hline Dicloreto de paraquate & 1,965 & $-4,662$ & 0,871 & $-0,850$ & $-0,989$ \\
\hline Difenoconazole & 0,020 & $-0,356$ & 0,392 & $-0,850$ & $-0,998$ \\
\hline Diuron & 0,144 & 1,933 & 0,206 & $-0,850$ & $-1,000$ \\
\hline Espiromesifeno & $-1,358$ & $-1,429$ & $-1,909$ & $-0,850$ & $-0,688$ \\
\hline Etefom & 1,667 & 0,030 & 0,325 & $-0,850$ & $-0,993$ \\
\hline Famaxadone & $-1,109$ & $-0,041$ & $-1,002$ & $-0,850$ & $-0,996$ \\
\hline Fenoxaprope-p-etílico & $-1,467$ & $-0,971$ & $-1,465$ & $-0,850$ & 0,176 \\
\hline Fentoato & $-0,224$ & 1,203 & $-1,395$ & $-0,850$ & $-0,999$ \\
\hline Fluazifop-P-butil & $-1,263$ & $-1,000$ & $-1,923$ & $-0,850$ & $-0,859$ \\
\hline Glifosato & 0,806 & 0,431 & 0,371 & $-0,850$ & $-0,996$ \\
\hline Hexazinone & 1,492 & 5,552 & 1,213 & 4,414 & $-1,000$ \\
\hline Imidacloprido & 1,043 & 4,165 & 2,157 & 2,083 & $-1,000$ \\
\hline Lambda-cialotrina & $-1,306$ & $-5,664$ & $-1,633$ & $-0,850$ & $-0,933$ \\
\hline Linurom & 0,189 & 1,704 & $-0,675$ & $-0,850$ & $-0,999$ \\
\hline Malathion & $-0,630$ & $-1,815$ & $-1,818$ & $-0,850$ & $-0,561$ \\
\hline Mancozebe & $-1,127$ & $-2,431$ & $-1,462$ & $-0,850$ & $-0,587$ \\
\hline Metalaxyl M & 0,923 & 1,074 & $-0,129$ & $-0,850$ & $-0,999$ \\
\hline Methomyl & 1,552 & 1,589 & 0,353 & $-0,850$ & $-1,000$ \\
\hline Paraquat & 2,316 & $-10,943$ & 0,839 & $-0,850$ & $-0,986$ \\
\hline Pencycurom & $-0,722$ & $-0,328$ & $-0,643$ & $-0,850$ & $-0,997$ \\
\hline Spinosad & 0,122 & $-1,887$ & 0,276 & $-0,850$ & $-0,898$ \\
\hline Picloram & 0,687 & 6,926 & 1,671 & 5,917 & $-1,000$ \\
\hline Tebuconazole & 0,072 & 1,575 & 0,133 & $-0,850$ & $-0,999$ \\
\hline Thiamethoxam & 1,385 & 4,465 & 2,056 & 2,609 & $-1,000$ \\
\hline Trifloxixtrobina & $-1,513$ & $-1,415$ & $-1,556$ & $-0,850$ & $-0,710$ \\
\hline
\end{tabular}

Tabela 04 - Análise de variância dos índices GSI, GUS, LIX, LEACH e RLPI padronizados

\begin{tabular}{ccccc}
\hline Fonte & DF & $\begin{array}{c}\text { Variável dependente: INS } \\
\text { Soma dos } \\
\text { quadrados }\end{array}$ & $\begin{array}{c}\text { Quadrado } \\
\text { médio }\end{array}$ & F value \\
\hline Modelo & & 19,14 & 4,78 & 1,13 \\
Erro & 4,00 & 740,01 & 4,22 & 0,34 \\
Total corrigido & 175,00 & 759,15 & & \\
\hline
\end{tabular}

o p-valor considerado foi de 0,05

\section{CONCLUSÕES}

A comparação entre os índices GSI, GUS, LIX, LEACH, RLPI indicaram que não há diferenças entre os seus resultados, ou seja, eles tendem a ter outputs de potencialidade de lixiviação semelhantes, cada um em sua escala. Todavia, os três primeiros são mais indicados para gerenciamento por serem mais fáceis no processo de tomada de decisão, uma vez que são classificados em classes de potencial lixiviação, ao contrário do RLPI e LEACH, que são relativos.

A hierarquização dos agrotóxicos no que tange à capacidade de lixiviar e contaminar os recursos hídricos subterrâneos alocou os ingredientes ativos picloram, hexazinone, clorantraniliprole, carbofurano, thiamethoxam, 2,4 D sal dimetilamina, imidacloprido, azoxistrobina, cartape, dicloreto de paraquate, diuron, 2,4 D, paraquate, methomyl, glifosato, tebuconazole, linurom, fentoato, methalaxyl M nas 15 primeiras colocações, indicando que devem ser evitados para a maior proteção da sub-bacia do baixo rio Natuba.

Por meio do método de CDFA, destacaram-se negativamente, pela sua capacidade de lixiviar e contaminar o meio ambiente, os agrotóxicos: 2,4 D sal dimetilamina, carbofurano, hexazinone, imidacloprido e thiamethoxam tendo em vista terem atendido aos quatro critérios de enquadramento do índice. Todos eles foram alocados na faixa de risco dos índices GSI, GUS, LIX, LEACH, RLPI, confirmando sua alta periculosidade para o meio ambiente.

Dessa forma, propõe-se que os agrotóxicos sinalizados pelo grande 
potencial de lixiviação participem de um processo de reavaliação, de modo que possam ser substituídos e a região em estudo passe a utilizar os ingredientes ativos que sejam menos propensos a contaminar os recursos hídricos subterrâneos, contribuindo para sua sustentabilidade ambiental.

\section{AGRADECIMENTOS}

O primeiro autor deste trabalho agradece ao Instituto Federal de Educação, Ciência e Tecnologia de Pernambuco (IFPE) pela oportunidade de cursar o Mestrado Profissional em Gestão Ambiental, por meio do qual esse artigo científico foi produzido.

\section{REFERÊNCIAS}

ARAÚJO FILHO, J. C.; BARBOSA NETO, M. V.; SILVA, C. B.; ARAÚJO, M. S. B.; MENEZES, J. B. Levantamento semidetalhado dos solos da bacia hidrográfica do rio Natuba, Pernambuco. Revista Brasileira de Geografia Física, v. 06, n. 03, p. 384-397, 2013.

ARMAS, E. D.; MONTEIRO, R. T. R.; AMÂNCIO, A. V.; CORREA, R. M. L.; GUERCIO, M. A. Uso de agrotóxicos em cana-de-açúcar na bacia do rio Corumbataí e o risco de poluição hídrica. Química Nova, v. 28, n. 06, p. 975-982, 2005.

BARBOSA NETO, M. V. B.; SILVA, C. B.; ARAÚJO FILHO, J. C.; ARAÚJO, M. S. B.; BRAGA, R. A. P. Uso da terra na bacia hidrográfica do rio Natuba, Pernambuco. Revista Brasileira de Geografia Física, v. 04, n. 05, p. 961-973, 2011.

BISHOP, K. C. Industry's perspective on agricultural chemicals in water supply and drainage. In: Proceedings "Toxic Substances in agricultural water supply and drainage: U.S Comitee of irrigation and drainage, 1986. Disponivel em: http://agris.fao.org/agrissearch/search.do?recordID=US8844824. Acesso em: 01 out. 2017.

BRAGA, R. A. P.; GUSMÃO, P. T. de; MESEL, M. S.. A Poluição do Rio Tapacurá: consequências e alternativas. Recife: Editora Universitária, 2006. 30 p.

BRITTO, F. B. Pesticidas no alto do rio Poxim e os riscos de contaminação. 2011. 105 f. Dissertação (Mestrado em Agroecossistemas), Universidade Federal de Sergipe, São Cristóvão, 2011.

CAVAlCANTI, N. A. O.; FeRREIRA, V.; NASCIMENTO, R. M. Proposta de cursos para capacitação dos agricultores como instrumentos de educação ambiental, bacia hidrográfica do Natuba, município de Vitória de Santo Antão - PE. In: CONNEPI. 5. 2010. Maceió. Anais... Maceió: IFAL, 2010. 5 p.

GUSTAFSON, D. I. Groundwater Ubiquity Score: a simple method for assessing pesticide leachability. Environmental Toxicology and Chemistry, v. 08, p. 339-357, 1989.

HORNSBY, A. G.; BUTTLER, T. M.; BROWN, R. B. Managing pesticides for crop production and water quality protection: practical grower guides. Agriculture, Ecosystems and Environment, v. 46, n. 1/4, p. 187-196, 1993.

LASKOWSKI, D. A.; GORING, C. A. I.; MCCALL, P. J.; SWANN, R. L. Terrestrial Environment. In: CONWAY, R. A. (Ed.) Environmental Risk Analysis for Chemicals. New York: Krieger Publishing Company, 1982. p. 198-240.

LEWIS, K. A.; TZILIVAKIS, J.; WARNER, D. J.; GREEN, A. An international database for pesticide risk assessments and management. Human and Ecological Risk Assessment: an international journal, v. 22, n. 04, p.10501064, 2016.

LONDRES, F. Agrotóxicos no Brasil: um guia para ação em defesa da via. Rio de Janeiro: AS-PTA, 2011. 190 p.

LOPES, M. M.; CASTELO BRANCO, V. T. F.; SOARES, J. B. Utilização dos testes estatísticos de Kolmogorov-Smirnov e Shapiro-Wilk para verificação da normalidade para materiais de pavimentação. Transportes, v.21, n.1., p. 59-66, 2013.
MARQUES, J. G. C. Proposições para o gerenciamento do uso de agrotóxicos utilizados na bacia hidrográfica do Natuba, Vitória de Santo Antão, Pernambuco. 125 p. 2017. Dissertação (Mestrado Profissional em Gestão Ambiental), Instituto Federal de Educação, Ciência e Tecnologia de Pernambuco, Recife, 2017.

MARQUES, J. G. C. M.; NASCIMENTO, R. M.; LYRA, M. R. C. C.; CARVALHO, R. M. C. M. O.; MONTENEGRO, S. M. G. L.; SILVA, J. A.; SLVA, J. C. O manejo de agrotóxicos por produtores rurais de hortaliças na sub bacia do Natuba, município de Vitória de Santo Antão - PE, Brasil. Cientec, v. 05, n. 01, p. 10-22, 2013.

MENEZES, J. B.. Levantamento das bases de dados da bacia do rio Natuba - PE: estudo de caso da Pedologia, Geomorfologia e Cobertura Vegetal. 2010. 67 f. Dissertação (Mestrado em Geografia), Universidade Federal de Pernambuco, Recife, 2010.

MENEZES, J. B.; ARAÚJO FILHO, J. C.; SILVA, C. B.; BARBOSA NETO, M. V.; ARAÚJO, M. S. B.; CAVALCANTI, L. C. S. Melhoria do mapeamento de solos da escala 1:100.000 para 1:25.000 com base em estudos morfológicos na bacia do rio Natuba. Revista de Geografia, v. 27, n. 03, p. 156-165, 2010.

NASCIMENTO, R. M. Impactos dos agrotóxicos na contaminação ambiental da produção de hortaliças no baixo rio Natuba, Pernambuco. 2013. 167 f. Tese (Doutorado em Engenharia Civil), Universidade Federal de Pernambuco, Recife, 2013.

NORONHA, C. R. B.; LIRA, E. B. S.; MORAIS, A. S. Agrochemicals and horticulturists: the invisible risks inserted in food produced in Natuba - Vitoria de Santo AntãoPE. Revista GEAMA, v. 01, n. 01, p. 93-103, 2016.

OLIVEIRA, M. C.; LIMA, L. A.; SILVA, A. C.; NASCIMENTO, J. M.; COLOMBO, A. Índice de GUS e GSI na avaliação da contaminação em águas subterrâneas por fungicidas na tomaticultura. In: CONGRESSO BRASILEIRO DE ÁGUAS SUBTERRÊNAS. 19. 2016. Campinas. Anais... São Paulo: ABAS, 2016a. 9 p.

OLIVEIRA, M. C.; LIMA, L. A.; SILVA, S. C.; NASCIMENTO, J. M.; COLOMBO, A Índice de GUS e GSI na avaliação da contaminação em águas subterrâneas por fungicidas da bataticultura. In: CONGRESSO BRASILEIRO DE ÁGUAS SUBTERRÂNEAS. 19., 2016. Campinas. Anais... Campinas: ABAS, 2016b. 8 p.

PFEIFFER, M. Groundwater Ubiquity Score. 2010. Disponível em: http://www.ptrpest.com/pdf/groundwater_ubiquity.pdf. Acesso em: 05 out 2018.

RIBEIRO, E. P. Eficácia no processo de devolução de embalagens de agrotóxicos vazias na comunidade do Natuba: área produtora de hortaliças de Vitória de Santo Antão - Pernambuco. 2011. 161 f. Dissertação (Mestrado em Gestão do Desenvolvimento Local Sustentável), Universidade de Pernambuco), Recife, 2011.

RODRÍGUEZ, J. M. Evaluation of groundwater quality and selected hydrologic conditions in the South Coast aquifer, Santa Isabel area, Puerto Rico. Virginia: U.S. Geological Survey, 2013.36 p.

RODRIGUES, J. E. C. Uso de agrotóxicos e seu impacto na saúde do trabaIhador rural no município de Vitória de Santo Antão - PE: um estudo de caso. 2006. 110 p. Dissertação (Mestrado em Tecnologia Ambiental) Instituto Tecnológico de Pernambuco, Recife, 2006.

SAATMAN, T. M. Avaliação da qualidade da água subterrânea em área de cultivo de hortaliça em relação à contaminação por agrotóxico. 2016. $51 \mathrm{f}$. Monografia (Graduação em Tecnologia em Gestão Ambiental), IFPE, Recife, 2016.

SILVA, C. B.; ARAÚJO, M. S. B.; ARAÚJO FILHO, J. C.; BITTAR, S. M. B. Bacia hidrográfica do rio Natuba - PE: uma análise através de parâmetros morfométricos. In: SIMPÓSIO BRASILEIRO DE GEOGRAFIA FÍSICA APLICADA. 8. 2017. Campinas. Anais... Campinas: Instituto de Geociências, 2017. p. 489-500.

SILVA, C. E. M. Programa de educação ambiental e proposta de pagamento por serviços ecossistêmicos no assentamento Chico Mendes (Ronda), microbacia do Alto Natuba, afluente do Tapacurá - Pombos - PE. 2007. Monografia (Graduação em Gestão Ambiental), Instituto Federal de Educação, Ciência e Tecnologia de Pernambuco, Recife, 2007. 
SOARES, D. F.; FARIA, A. M.; ROSA, A. H.. Análise de risco de contaminação de águas subterrâneas por resíduos de agrotóxicos no município de Campo Novo do Parecis (MT), Brasil. Engenharia Sanitária e Ambiental, v. 22, n 02, p. 277-284, 2017.

SOUZA, D.; GREEN, V. Avaliação de Estudos de Vulnerabilidade e Adaptação à Mudança do Clima nas cidades brasileiras. In: TEIXEIRA, B. S.; ORSINI, J. A. M.; CRUZ, M. R. (Ed.). Modelagem climática e vulnerabilidades setoriais à mudança do clima no Brasil. Brasília: Ministério de Ciência, Tecnologia e Inovação, 2016. p.511-589.
SOUZA, S. F.; ARAÚJO, M. S. B. Avaliação da cobertura vegetal densa na sub bacia do rio Natuba do estado de Pernambuco. In: SIMPÓSIO BRASILEIRO DE SENSORIAMENTO REMOTO. 15. 2011. Curitiba. Anais... São José dos Campos: INPE, 2011. p. 7753-7760.

SPADOTTO, C. A. Screening Method for Assessing Pesticide Leaching Potential. Pesticidas: Revista de Ecotoxicologia e Meio Ambiente, v. 12, p. 69-78, 2002.

WILKERSON, M. R.; KIM, K. D. The pesticide contamination prevention act: setting specific numeral values. California: California Departament of Food and Agriculture - CDFA, 1986. 29 p. 